\title{
交联液晶聚合物光致形变及其柔性器件
}

顾伟 ${ }^{(1)}$, 卿釱 ${ }^{(1)}$, 韦嘉 ${ }^{(1)}$, 俞燕蕾 ${ }^{(12 *}$

(1) 复旦大学材料科学系, 上海 200433;

(2) 复旦大学聚合物分子工程国家重点实验室, 上海 200433

*联系人, E-mail: ylyu@fudan.edu.cn

2016-01-05 收稿, 2016-03-01 修回, 2016-03-02 接受, 2016-04-07 网络版发表

国家自然科学基金 $(51225304,21273048,21134003,51573029)$ 和上海市优秀学术带头人计划(15XD1500600)资助

摘要光响应聚合物材料是指能够在光的作用下发生某些化学或物理反应, 产生一系列结构和形态变化的功能 聚合物材料. 在光响应聚合物体系中引入液晶基元通过适度交联可形成光响应交联液晶聚合物，它结合了液晶的 各向异性以及聚合物网络的橡胶弹性, 具有优异的协同作用. 且因为光能具有环保性、远程可控性、瞬时性等优 异的特性, 光响应交联液晶聚合物备受关注. 通过合理的设计, 光响应交联液晶聚合物可以实现全光驱动的形变, 并且可以制成多种柔性智能执行器, 在人工肌肉、微型机器人、微泉、传感器等仿生和智能微机械系统领域有着 广泛的应用前景。本文综述了光响应交联液晶聚合物近年来的研究进展, 包括其二维和三维形变、微观形变引起 的表面形貌或其他性质的变化以及基于形变制成的柔性智能执行器，阐述了光响应的机理，并展望了该领域的发 展前景.

关键词光响应, 交联液晶聚合物, 执行器, 形变, 偶氮苯

交联液晶聚合物是液晶聚合物通过适度交联形 成的液晶网络, 兼具液晶的各向异性以及聚合物网 络的橡胶弹性, 具有优异的协同作用 ${ }^{[1 \sim 3]}$. 它能够在 外场(热、电、磁、光等)的刺激下, 通过改变液晶基 元的排列而产生各向异性的形状(尺寸)变化 ${ }^{[4 \sim 6]}$. 与 众多刺激方式相比, 光驱动方式由于其可控性好、可 远程操作、对使用环境无要求而受到较多关注. 光响 应交联液晶聚合物通常含有能吸收光能的分子或官 能团, 在光的作用下会发生某些化学或物理反应, 产 生一系列结构和形态变化, 从而表现出特定的功能. 偶氮苯类的衍生物是目前研究最为广泛的一类光响 应基团, 在光的作用下, 偶氮苯可以实现顺反异构化 的改变. 其反式构象在热力学上处于稳定状态, 在紫 外光的照射下, 反式的偶氮苯发生异构反应, 转变成 顺式偶氮苯; 顺式偶氮苯构象热力学上处于非稳定
状态, 自然状态下可逐渐恢复到反式构象, 经可见光 的照射或者加热也可使顺式偶氮苯回复到反式结 构 $^{[7]}$. 偶氮苯的异构化可导致交联液晶聚合物发生光 化学相转变和光取向过程, 从而引起形变.

\section{1 光响应原理}

\section{1 光化学相转变}

液晶由于其协同作用，当少量分子的排列在外 部刺激下发生变化时, 其他液晶分子的排列方向也 会发生改变，因此改变整个体系所需的能量很少(仅 需改变 $1 \%$ (摩尔比)液晶分子排列方向的能量 $)^{[5]}$. 在 液晶体系中引人光敏分子或基团, 经过光照光敏分 子发生光化学反应可引起液晶相态的变化 ${ }^{[8 \sim 10]}$. 以偶 氮苯类液晶体系为例，反式的偶氮苯分子呈棒状结 
构，形状与其他液晶分子相似，对整个液晶体系有着 稳定化作用, 而顺式的偶氮苯分子则是弯曲结构, 倾 向于使整个液晶体系取向发生紊乱, 如图1(a)所示. 因此, 含顺式偶氮苯的液晶体系从液晶相到各向同 性相的相转变温度 $T_{\mathrm{cc}}$ 要低于含反式偶氮苯相转变温 度 $T_{\mathrm{ct}}$ (图 1(b)). 若样品温度 $T$ 在 $T_{\mathrm{cc}}$ 和 $T_{\mathrm{ct}}$ 之间, 用光照 引起偶氮苯反式到顺式异构化反应, 样品的相转变 温度 $T_{\mathrm{c}}$ 就会随着体系中顺式偶氮苯的增多而降低. 当 $T_{\mathrm{c}}$ 小于 $T$ 时, 样品就会由液晶相转为各向同性相 ${ }^{[5]}$. 光化学反应通常是可逆的, 偶氮苯顺式到反式的异 构化反应会诱导样品回到液晶相, 因而液晶体系的 相转变可在等温情况下由光响应分子或基团的光化 学反应引发. 当含有偶氮苯的交联液晶聚合物接受 紫外光照射时, 分子取向的变化将进一步使整个交 联网络产生各向异性的宏观形变. 这种由于液晶体 系的相变所产生的形变一般都是双向可控的, 很大 程度上拓展了材料的应用范围.

\section{2 光取向}

光响应型分子或基团要吸收光到达激发态, 需 满足一定条件, 即分子跃迁矩的方向和有效光电场 矢量方向一致. 而反式偶氮苯 $\pi-\pi^{*}$ 的跃迁矩和分子长 轴方向平行, 因此偶氮苯对有效光的吸收依赖于分 (a)

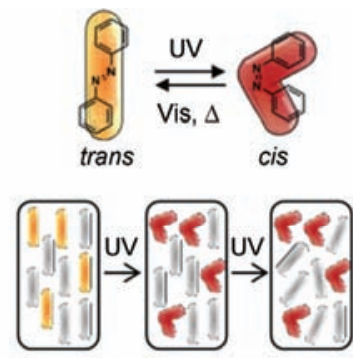

(c)

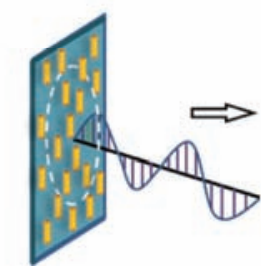

(b)

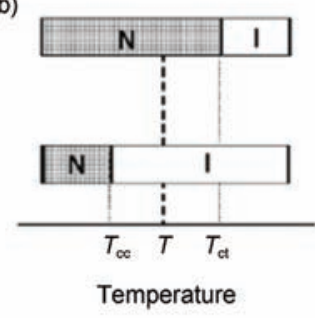

图 1 (网络版彩色)(a), (b) 偶氮苯液晶体系的光化学相转变示意图 $(\mathrm{N} \text { : 向列相; I: 各向同性相 })^{[5,7]}$; (c) 非线性偏振光对含有偶氮苯的液 晶聚合物光取向示意图

Figure 1 (Color online) (a), (b) Schematic representation of photochemical phase transition in the azobenzene-containing liquid crystal systems (N: nematic; I: isotropic) ${ }^{[5,7]}$. (c) Photoalignment of azobenzenecontaining liquid crystal polymers with linearly unpolarized light
子长轴与光的电场矢量方向的夹角. 根据Weigert效 应, 偶氮苯可由光进行三维取向, 当反式偶氮苯跃迁 矩(长轴)方向和线性偏振光偏振方向平行时, 偶氮苯 可吸收能量转变为激发态然后发生反式-顺式异构化 过程; 而当反式偶氮苯分子跃迁矩(长轴)方向和线性 偏振光偏振方向垂直时, 偶氮苯不会吸收光能, 因此 不会发生异构化反应. 经过反复的反式-顺式-反式的 循环, 一旦反式偶氮苯跃迁矩和光偏振方向垂直, 对 人射光的照射就呈惰性, 再不会反生异构化反应 ${ }^{[11]}$. 也就是说, 经过多次异构化循环过程, 最终所有的偶 氮苯分子都会垂直于光偏振方向排列，这种取向可 通过加热或者光化学反应“擦除”, 然后继续用线性 偏振光照射可重新取向. 当用非线性偏振光照射含 有偶氮苯的聚合物时, 偶氮苯会沿着光源前进方向 取向. 这是因为只有光源前进的方向才和光的电场 矢量方向垂直, 偶氮苯的长轴沿着此方向排列时不 会吸收光能, 因而最终偶氮苯分子倾向于沿此方向 排列, 如图1(c)所示. 当含有偶氮苯的交联液晶聚合 物发生光再取向过程时, 偶氮苯排列发生变化也可 导致交联液晶聚合物发生形变.

例如, Bunning课题组 ${ }^{[12 ~ 15]}$ 制备出含有偶氮苯的 交联液晶聚合物悬臂梁, 用多波长 $(457,488,514 \mathrm{~nm})$ 氩离子偏振激光照射, 发现悬臂梁可产生频率高达 $270 \mathrm{~Hz}$ 的摆动 ${ }^{[15]}$. 这是由于在偏振激光的照射下, 偶 氮苯液晶基元由平行于激光的偏振方向排列转变为 垂直于偏振方向排列 (Weigert效应), 在悬臂梁摆动 的过程中上下表面交替产生变化而产生表面收缩, 从而使悬臂梁快速摆动.

目前, 对于交联液晶聚合物光致形变的研究主 要是基于光化学相转变原理, 因此, 接下来的论述据 此展开.

\section{2 交联液晶聚合物光致形变行为}

\section{1 光致二维形变}

光响应性基团的引人可使交联液晶聚合物发生 二维的形变, 比如膨胀和收缩. 偶氮苯在从反式异构 体转变成顺式异构体的过程中, 分子的长度由 $9 \AA$ 缩 短为 $5.5 \AA$, 这种分子构型的转变可引起交联液晶聚 合物大的收缩 ${ }^{[9]}$. 2001年, Finkelmann等人 ${ }^{[16]}$ 将偶氮 苯基团引人到以聚硅氧烷为主链的单畴向列相交联 液晶聚合物中，偶氮苯基团既是光发色团又是液晶 
基元, 在紫外光照射下, 偶氮苯发生光致异构, $\mathrm{V}$ 字 型的顺式异构体将向列相液晶体系打乱, 由于液晶 基元与聚合物主链交联形成三维的网络结构, 偶氮 苯基团与主链的偶合作用使交联液晶聚合物沿着液 晶基元排列方向发生收缩形变, 形变量达到 $20 \%$. Li 等人 ${ }^{[17]}$ 通过光(近红外光)聚合合成了含有偶氮苯基 才的腰挂型交联液晶聚合物薄膜, 该薄膜在紫外光 的照射下快速收缩(小于 $1 \mathrm{~min}$ ), 收缩率可达 $18 \%$.

\section{2 光致三维形变}

尽管交联液晶聚合物表现出优异的二维运动特 性, 但是各种形式的三维运动(弯曲、扭曲、旋转)在 实际应用中更具价值. Ikeda课题组 ${ }^{[18,19]}$ 报道了含有 偶氮苯的单畴液晶薄膜的光致弯曲行为, 他们发现 单畴交联液晶聚合物薄膜在紫外光照射下会沿着液 晶基元的排列方向向着人射光发生弯曲, 用可见光 照射后薄膜恢复到最初的平展状态. 这是因为在 360 $\mathrm{nm}$ 附近, 偶氮苯的摩尔吸光系数较高 $\left(\right.$ 约为 $2.6 \times 10^{4}$ $\mathrm{L} /(\mathrm{mol} \mathrm{cm})), 99 \%$ 的光子都被薄膜厚度方向 $1 \mu \mathrm{m}$ 内的 偶氮苯吸收, 也即只有薄膜的表层发生了光致收缩, 而薄膜其余部分因为基本上没有感应到紫外光而没 有发生任何变化, 因此薄膜在内应力的驱动下发生 了弯曲. 当弯曲的薄膜受到可见光照射后, 顺式偶氮 苯回复到反式状态, 交联液晶聚合物表面的液晶基 元恢复有序排列而使收缩层恢复到原来的长度, 所 以薄膜又回复到原来的平整状态. 他们还用热聚合 的方法制备了一种多畴的交联液晶聚合物薄膜, 偶 氮苯液晶基元在微观的畴内形成有序排列, 而宏观 上则是无序状态, 由于长棒状的偶氮苯液晶基元对 线性偏振光的吸收很大程度上与偏振光的偏振方向 有关, 因此, 当用线性偏振紫外光照射多畴的交联液 晶聚合物薄膜时, 液晶基元排列方向与线性偏振光 偏振方向一致的畴内发生光致收缩, 于是整个薄膜 沿着偏振光的偏振方向发生了弯曲形变, 通过改变 人射光偏振方向的方法即可简单地实现对薄膜弯曲 方向的精确控制 ${ }^{[20]}$.

另外, 液晶基元的排列方向对交联液晶聚合物 的形变方式有很大影响. Ikeda课题组 ${ }^{[21]}$ 发现垂直取 向的交联液晶聚合物薄膜在紫外光的照射下表现出 不同的光致弯曲行为. 平行取向的交联液晶聚合物 薄膜在紫外光的照射下表面发生收缩, 朝光源方向 弯曲, 而具有垂直取向的交联液晶聚合物的表面则
发生膨胀, 发生远离光源方向的弯曲形变. Ikeda课题 组 ${ }^{[22]}$ 还利用光聚合技术制备了高度有序并且玻璃化 转变温度 $\left(T_{\mathrm{g}}\right)$ 低的铁电交联液晶聚合物薄膜, 并且发 现铁电液晶由于其响应速度快、液晶基元排列高度有 序, 交联液晶聚合物薄膜光响应性更优越. 薄膜在光 强为 $280 \mathrm{~mW} / \mathrm{cm}^{2}$ 的氩离子激光 $(364 \mathrm{~nm})$ 照射下在毫 秒级时间内完成了整个弯曲过程, 展现了极快的光 响应速度.

光致形变交联液晶聚合物大多是由紫外光驱动 的, 然而从实际应用的角度来看, 由于紫外光的强扩 散效应, 其在生物组织上的穿透能力受到限制, 并且 紫外光对细胞和组织都有严重的危害, 所以开发能 够可见光驱动的交联液晶聚合物材料具有很大的意 义. 本课题组 ${ }^{[23,24]}$ 在偶氮苯基团上连接苯乙炔基形 成长共轭结构的偶氮二苯乙炔生色团, 使材料的最 大吸收产生红移，因而开发出了可见光(甚至是太阳 光)直接驱动的光致弯曲新材料. 其弯曲机理与紫外 光驱动的交联液晶聚合物薄膜弯曲机理类似, 薄膜 表层的偶氮二苯乙炔基团在430 $\mathrm{nm}$ 光照后发生异构 化, 表层发生收缩, 导致薄膜向着人射光的方向弯 曲. 其驱动波长大于 $430 \mathrm{~nm}$, 实现了太阳能到机械能 的直接转换. 本课题组 ${ }^{[25]}$ 还成功地将稀土上转换发 光纳米粒子与含有偶氮二苯乙炔的交联液晶聚合物 复合, 得到在连续波980 nm近红外光照射下可产生 快速弯曲形变的薄膜. 在连续波 $980 \mathrm{~nm}$ 的照射下, 稀 土上转换发光纳米粒子在 450 和 $475 \mathrm{~nm}$ 处有发射峰, 与偶氮二苯乙炔的吸收带匹配, 因此由上转换发光 纳米粒子发出的上转换光可使偶氮二苯乙炔基团发 生顺反异构化反应, 并且进一步诱导液晶基元排列 发生改变, 最终引起薄膜宏观形变. 之后, 基于三线 态-三线态湮灭(TTA)上转换发光技术, 本课题组 ${ }^{[26]}$ 又开发出低功率635 $\mathrm{nm}$ 红光激发的光致形变交联液 晶聚合物/聚氨酯复合薄膜, 如图2(a)所示, 并且该薄 膜在透过生物组织的红光照射下仍具有响应性(图 2(b)).

\section{3 光致表面性质变化}

二维和三维形变是交联液晶聚合物光致形变的 一个重要表现形式, 交联液晶聚合物在光的刺激下 发生微观形变从而引起表面形貌发生改变也是研究 的热点 ${ }^{[27,28]}$. Liu和Broer ${ }^{[29]}$ 将液晶单体和光引发剂的 混合物旋涂在含垂直聚酰亚胺取向层的玻璃基片上, 

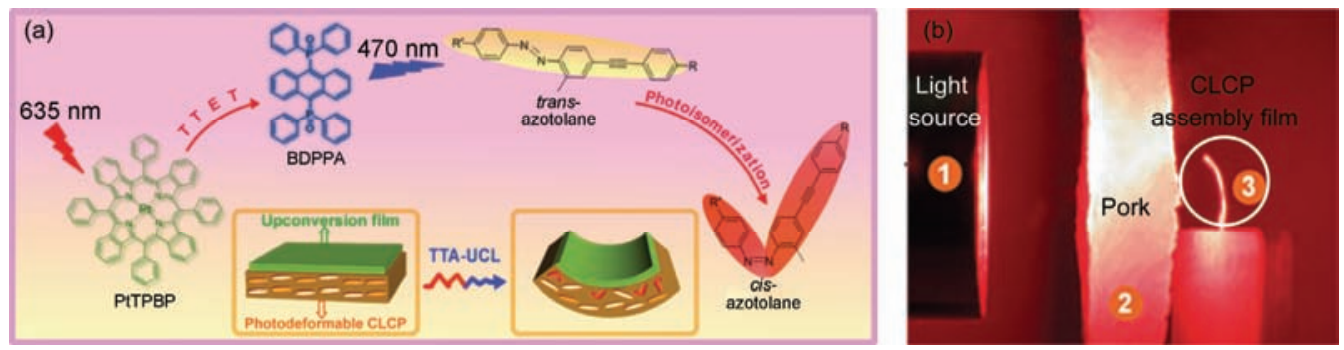

图 2 (网络版彩色)(a) TTA上转换材料PtTPBP(敏化剂)和BDPPA(湮灭剂)化学结构式及上转换发光激发交联液晶聚合物复合膜弯曲示意图; (b) 交联液晶聚合物复合薄膜在透过生物组织的红光照射下形变照片 ${ }^{[26]}$

Figure 2 (Color online) (a) Illustrations of the deformation of the two layer assembly film induced by TTA-based upconversion luminescence. Chemical structures of TTA system composed of PtTPBP (sensitizer) and BDPPA (annihilator) are also shown. (b) Photographs of assembly film bending toward the light source in response to the incident light transmitting through the pork ${ }^{[26]}$

由于混合物中含有手性分子, 液晶基元自发排列成 螺旋结构(螺旋轴平行于玻璃基板). 聚合后的交联液 晶聚合物薄膜表面平整, 经紫外光照射偶氮苯发生 反式到顺式的异构化过程, 如图3(a)所示, 螺旋结构 中平行排列的部分形成凸起, 而垂直排列的部分形 成凹槽, 由此交联液晶聚合物薄膜表面粗䊁度发生 改变. 测试结果表明, 紫外光照射后薄膜表面摩擦因 数从光照前的0.4 0.5减小为 0.1 , 利用这一特性, Liu 和Broer ${ }^{[29]}$ 构建了图3(b) 所示的夹具可实现对物体的

(a)

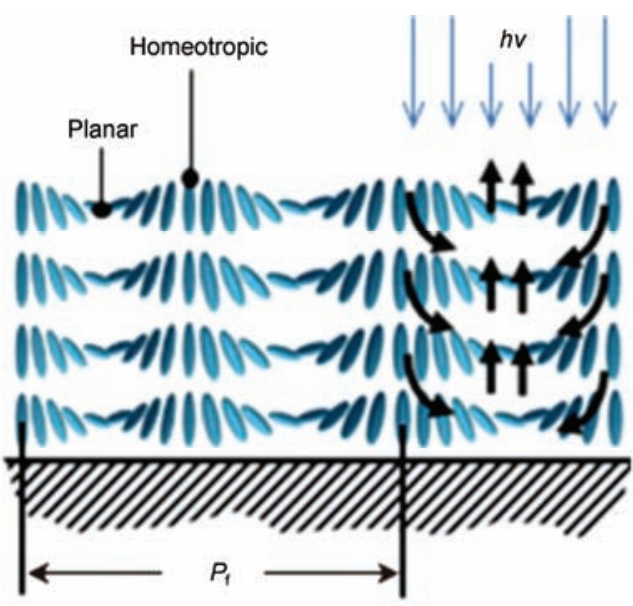

(b)

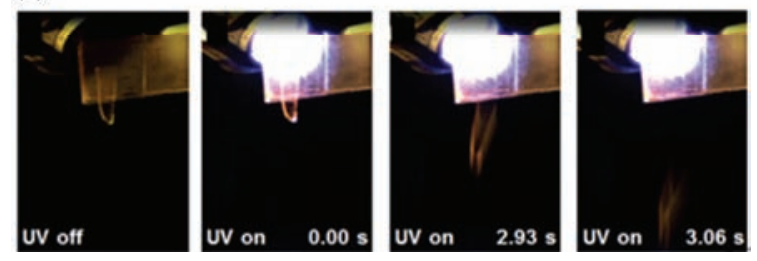

图 3 (网络版彩色)(a) 液晶基元排列示意图; (b) 交联液晶聚合物薄 膜夹具对物体的自由释放 ${ }^{[29]}$

Figure 3 (Color online) (a) Schematic representation of cholesteric liquid-crystal networks and the dynamics of the fingerprints. (b) Snapshots of a gripper that releases an object upon UV illumination ${ }^{[29]}$
自由释放, 可用在微型机器人指尖.

交联液晶聚合物的微观形变除了可以引起表面 形貌及粗糙度的变化, 还可引起表面黏附性的改变. 本课题组 ${ }^{[30]}$ 通过二次复形法制备了具有微米级阵列 化表面含有偶氮苯的交联液晶聚合物的薄膜, 如图 4(a)所示. 该薄膜具有超疏水的性质, 通过紫外光/可 见光照射, 黏附性发生变化. 在光照前水滴可从薄膜 表面自由滚落, 经紫外光照射后水滴固定在表面; 继 续经可见光照射, 又可恢复到滚落状态(图4(c)).

含有偶氮苯的微阵列化交联液晶聚合物表面不 仅可用光控改变表面的黏附性, 微柱在紫外光照射 下沿长轴方向的收缩以及短轴方向的膨胀导致的微 阵列规整度的变化也可用于反射光谱的调控 ${ }^{[31]}$. 然 而, 此二维光子晶体反射峰强度较低, 本课题组 ${ }^{[32]}$ 制备了含有偶氮苯的交联液晶聚合物反蛋白石薄膜 (三维光子晶体), 制备方法如图 5(a)所示, 得到的薄 膜可反射677 $\mathrm{nm}$ 左右的可见光. 在紫外光照射下偶 氮苯发生异构化反应, 多畴的交联液晶聚合物反蛋 白石薄膜中孔结构的有序度降低, 引起光子禁带反 射峰强度的下降; 之后经可见光的照射, 反射光强度 回复(图 5(b)). 同时, 该薄膜还具有热响应性, 升温 到 $90{ }^{\circ} \mathrm{C}$ 反射峰几乎消失; 降温后反射峰又回复(图 $5(\mathrm{c}))$.

\section{4 柔性智能执行器}

光响应交联液晶聚合物除了可完成上述简单的 二维伸缩、三维弯曲运动, 还可通过合理设计制成多 种智能柔性执行器, 完成复杂形变, 在人工肌肉、微 型机器人、微泵、微阀等领域有广泛应用前景. Ikeda 课题组 ${ }^{[33]}$ 制备了一条传动履带, 该履带是由偶氮苯 交联液晶聚合物与柔性聚乙烯薄膜复合材料首尾相 
(a)

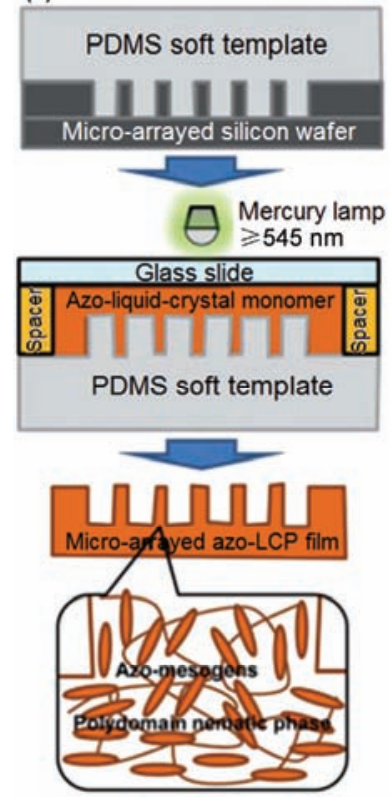

(b)
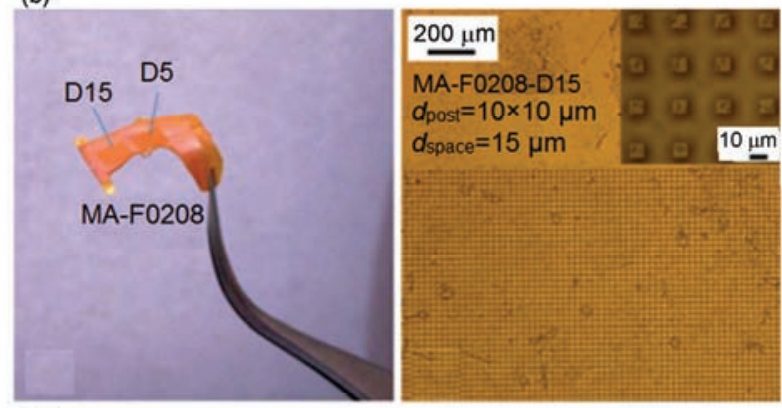

(c)

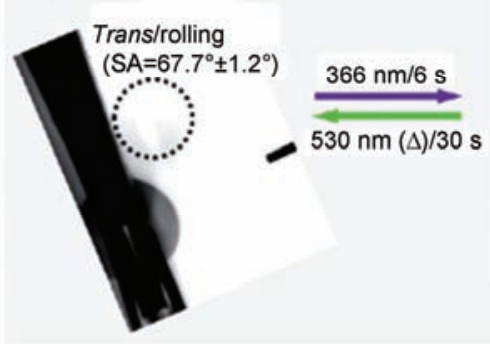

Cis/pinned $\left(\mathrm{S} A \geqslant 90^{\circ}\right)$

图 4 (网络版彩色)(a) 交联液晶聚合物微阵列薄膜制备示意图; (b) 图案化交联液晶聚合物薄膜的光学照片以及显微镜照片; (c) 交联液晶聚 合物光控黏附性变化水滴固定和滚落照片 ${ }^{[30]}$

Figure 4 (Color online) (a) Schematic illustration of the preparation process; (b) Optical photo and microscopic image of the CLCP film with patterned areas. (c) Microscopic profiles of the rolling and pinned water droplets ${ }^{[30]}$

(a)

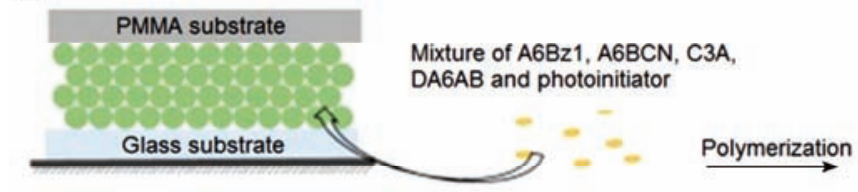

$\mathrm{Hg}$ lamp $\wedge>540 \mathrm{~nm}$
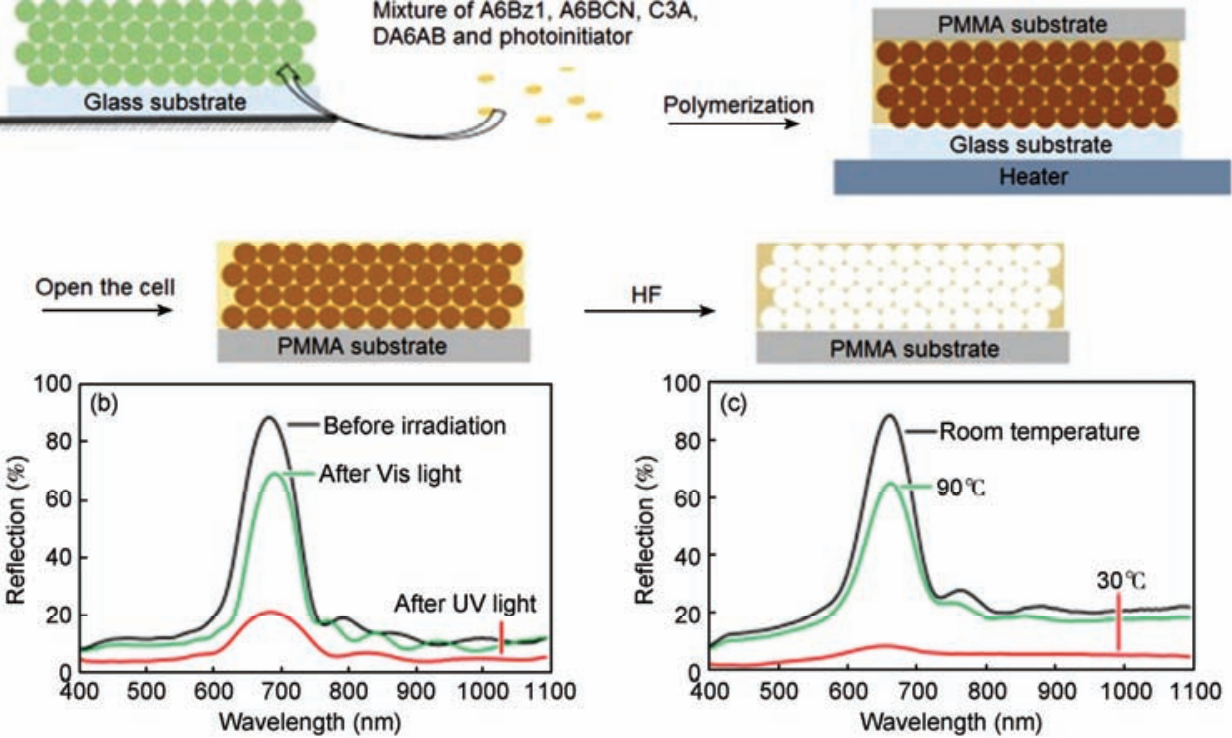

图 5 (网络版彩色)(a) 含偶氮苯交联液晶聚合物反蛋白石薄膜制备过程示意图; (b) 紫外光可见光照射前后薄膜反射光谱; (c) 薄膜反射光谱 随温度变化图 ${ }^{[32]}$

Figure 5 (Color online) (a) The fabrication process of the CLCP inverse opal film. (b) Reflection spectra of the azobenzene CLCP inverse opal film under UV light irradiation and subsequent visible light irradiation. (c) Reflection spectra of the inverse opal film as a function of temperature ${ }^{[32]}$

接而成. 分别用紫外光和可见光同时照射履带的右 上方和左上方，右侧的滑轮上产生一个收缩应力使
之逆时针转动, 而左侧的滑轮上产生一个膨胀的应 力也使其逆时针转动，于是整条履带便沿着逆时针 
方向开始转动, 而且这样的转动又会带来新的履带 接受紫外光和可见光的照射, 转动循环下去使得马 达能够持续旋转, 履带具体的结构和光致转动过程 如图 6(a) 和 (b) 所示. Ikeda课题组 ${ }^{[34]}$ 还利用该类交联 液晶聚合物与聚乙烯的复合薄膜实现了更为丰富的 三维运动. 他们将弯曲状复合薄膜的两端设计成摩 擦性不同的节点, 在紫外光和可见光的交替照射作 用下, 复合薄膜发生弯曲和回复的交替运动, 由于两 端摩擦性不同薄膜便如爬虫般向一个方向行走(图 6(c)). 这种复合薄膜还可通过紫外光和可见光照射 不同点实现机器人手臂般的运动, 如图6(d)所示.

本课题组 ${ }^{[35]}$ 利用含有偶氮二苯乙炔的交联液晶 聚合物薄膜与聚乙烯薄膜复合, 设计出具有 “手指”、 “手腕”、“手臂”等部件的柔性微机器人, 其各部分的 弯曲形变均由可见光驱动. 如图7(a)所示, 柔性微机 器人在移动物体时, 首先光源照射手指结构使其张 开; 接着照向手腕部位, 手腕部位的形变带动手指逐 渐靠向物体; 停止照射后, 手指逐渐恢复环状结构将 物体包住; 这时再照射上部的手臂结构, 其弯曲形变 带动整条手臂发生位移, 从而将物体移至目的地; 再 次照射手指部分, 环状结构打开, 将物体放下. 该微 型机器人可在大约 $45 \mathrm{~s}$ 内搬运约 $10 \mathrm{mg}$ 的物体, 达实
际驱动部件重量的10倍之多, 物体在水平方向被移 动了约 $20 \mathrm{~mm}$, 被“拎起”的最大高度约为 $5 \mathrm{~mm}$.

$\mathrm{Zhu}$ 与 $\mathrm{Yu}$ 等人 ${ }^{[36,37]}$ 合作制备了光驱动微洜, 微洜 结构设计图如图7(b)所示. 微泵主要由泵膜、泵腔、 液晶聚合物薄膜和盖板等组成, 当紫外线照射时, 交 联液晶聚合物薄膜表层收缩，向下弯曲，使得泵膜向 下变形, 泵腔体积减小压力增大, 从而人口阀关闭, 出口阀打开, 流体从泵腔流人出口管道 ${ }^{[36]}$. 当可见 光照射时, 光致弯曲材料从弯曲状态回复到平整状 态, 使得泵膜也向上运动, 回复到初始状态, 泵腔体 积增大压力减小, 从而人口阀打开, 出口阀关闭, 流 体从人口管道流人原腔. 如此不断循环, 从而实现流 体的单向驱动.

van Oosten等人 ${ }^{[38]}$ 用喷墨打印技术制备出了类 似纤毛功能的微型执行器. 该微纤毛的根部和顶部 分别含有对 360 和 $490 \mathrm{~nm}$ 的光有响应的液晶基元，通 过选用不同波长的光照射微纤毛执行器可实现不同 幅度的形变从而模拟纤毛的运动. 如图 8 所示, 利用 $490 \mathrm{~nm}$ 可见光照射时, 纤毛顶部产生微小弯曲, 根部 没有响应; 然后利用两种波长的光同时照射时, 根部 与顶部同时产生了响应，在整个长度范围产生大的 形变; 继续利用紫外光照射时, 纤毛的根部弯曲, 纤

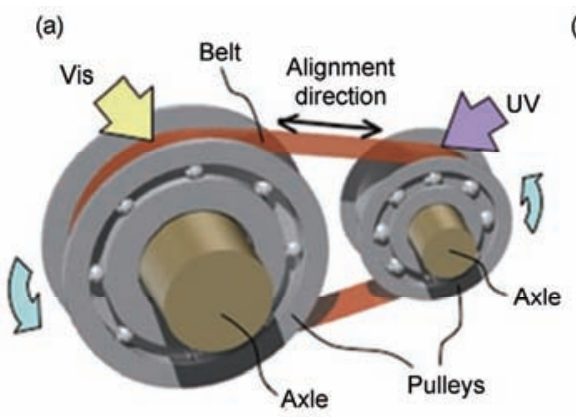

(b)
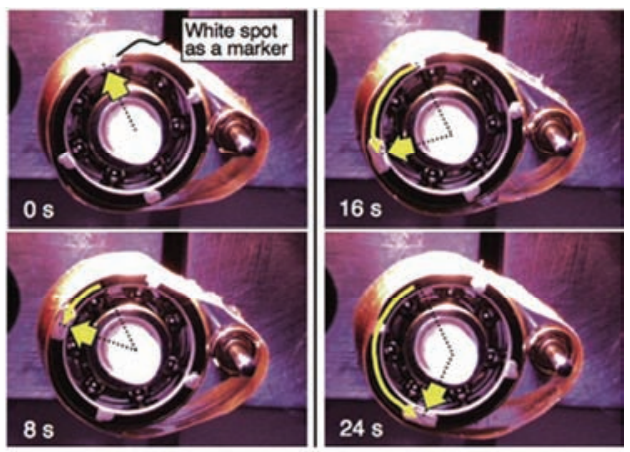

(c)
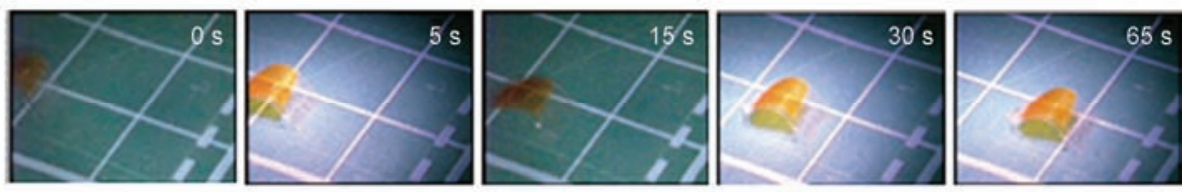

(d)
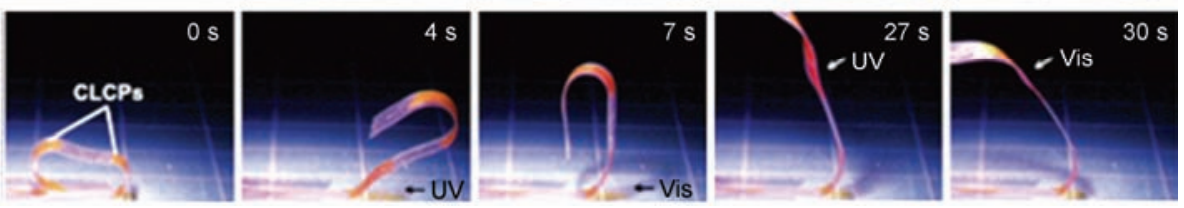

图 6 (网络版彩色)(a) 光驱动马达结构示意图; (b) 光照下马达转动图片 ${ }^{[33]}$; (c) 光驱动爬虫走动; (d) 光驱动机器人手臂运动 ${ }^{[34]}$

Figure 6 (Color online) (a) Schematic illustration of the light-driven motor. (b) Photographs showing the rotation of the motor operated by light exposure $^{[33]}$. The photoinduced inchworm walk (c) and the flexible robotic arm motion $(\mathrm{d})^{[34]}$ 
(a)
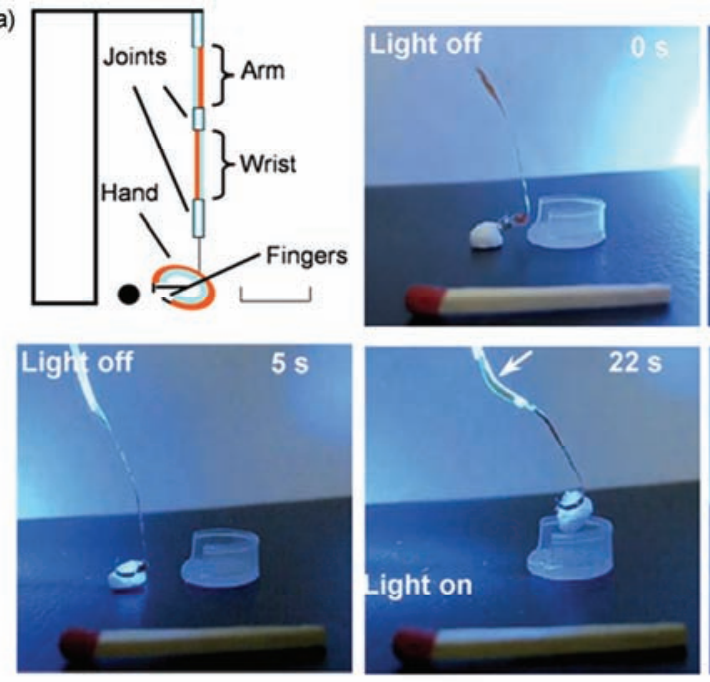
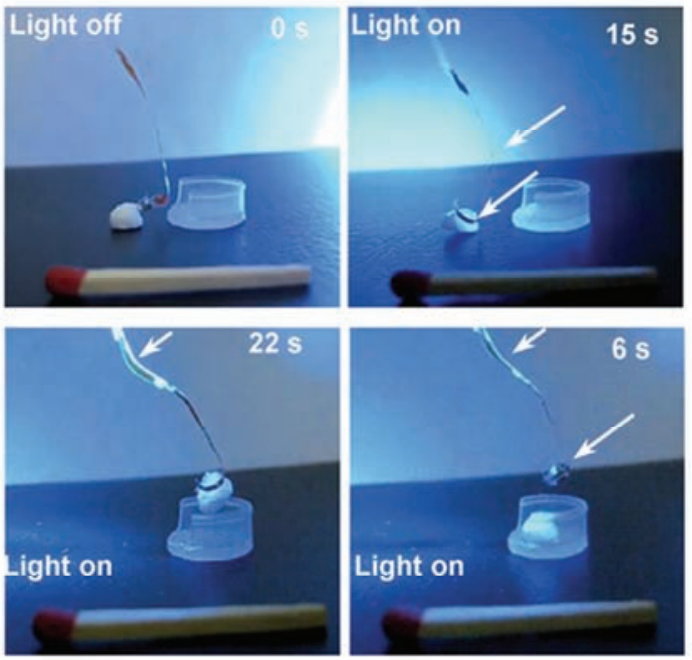

(b)
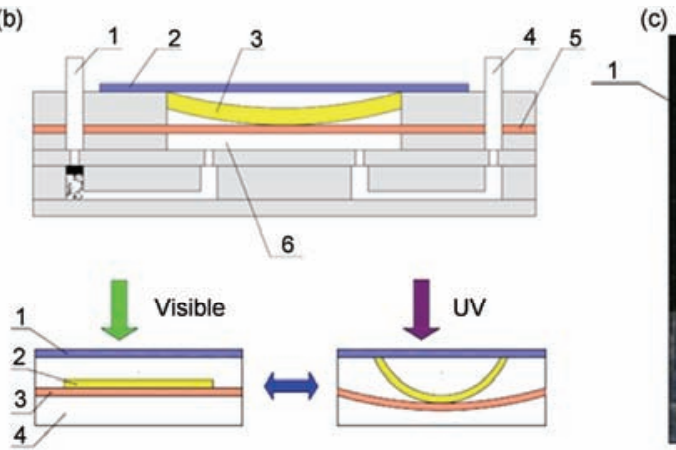

c)

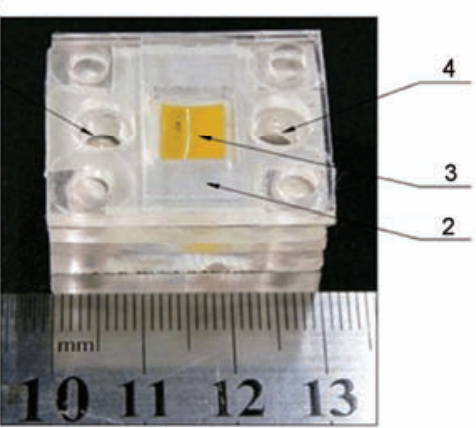

图 7 (网络版彩色)(a) 完全由可见光控制的微机器人动作实验照片及示意图 ${ }^{[3]}$; 光驱动微泵模拟(b)及实物图(c) ${ }^{[36]}$. 1. 人口; 2. 盖板; 3. 光致 弯曲材料; 4. 出口; 5. 泵膜; 6. 泵腔

Figure 7 (Color online) (a) Schematic illustration and photographs showing the microrobot manipulating the object ${ }^{[35]}$. (b) The section of the assembled prototype. (c) Photo of the experimental prototype ${ }^{[36]}$. 1. inlet, 2. press plate, 3. photodeformable material, 4. outlet, 5. pump membrane, 6. pump chamber

(a)
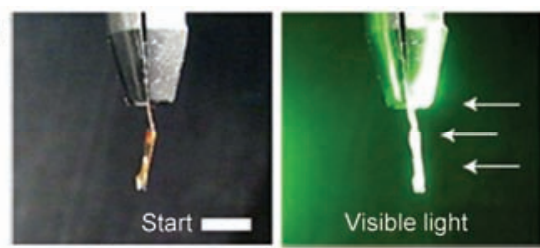

(b)

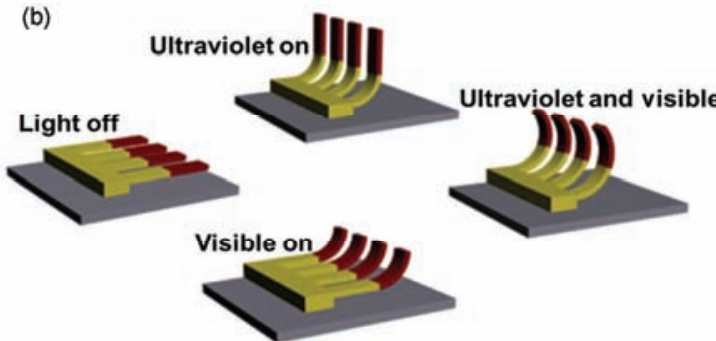

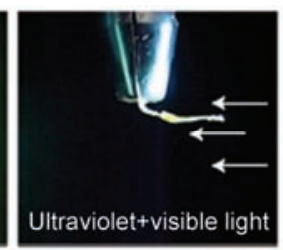
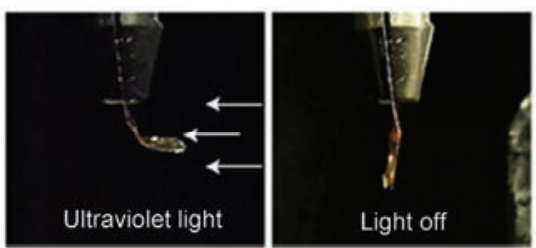

(c)
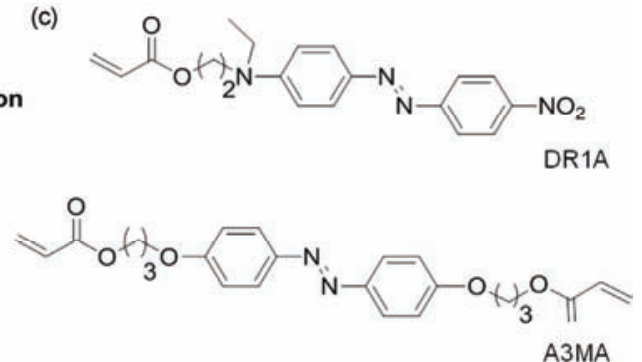

图 8 (网络版彩色)(a) 不同波长的光照下人工纤毛产生不对称运动; (b) 不同波长光照下微纤不对称运动示意图; (c) 对不同波长光响应的液 晶单体化学结构式 ${ }^{[38]}$

Figure 8 (Color online) (a) Steady-state responses of a liquid-crystal network actuator to different colours of light. (b) Artificial light-driven cilia produce an asymmetric motion controlled by the spectral composition of the light. (c) Chemical structures of the monomers used to form the actuators in this study ${ }^{[38]}$ 
毛顶端弯曲回复; 撤去光源, 微纤毛整个长度方向形 变回复. 这种器件的制备是可以选择不同的喷涂液 进行喷涂打印, 成本较低, 有利于大面积的制备响应 性的执行器件, 将来有望替代传统的电驱动执行器.

Fletcher课题组 ${ }^{[39]}$ 受到大自然界攀藤植物的启 发, 设计合成并研究了一种新型的类似弹簧的光响 应液晶弹性软执行器. 由于手性分子的存在, 液晶分 子在薄膜内部排列从上到下发生 $90^{\circ}$ 的扭曲变化. 当 沿不同的角度 $\varphi$ 剪切时, 得到丝带的形变方式也会不 同. 对于化学组成相同的薄膜, 仅通过改变裁剪就可 实现紫外光照下的伸展、收缩和螺旋翻转等(图9(b)). 当 $\varphi$ 为 $45^{\circ}$ 时, 薄膜外表面液晶基元垂直于长轴排列, 内表面液晶基元平行于长轴方向排列, 紫外光照射 时偶氮苯从棒状的反式异构体转变成 $\mathrm{V}$ 型顺式异构 体, 外表面沿长轴方向膨胀而内表面收缩, 从而弹簧 收缩(图9(c)). 随裁剪角度的不同(图9(d)), 薄膜可发 生伸展、扭曲和螺旋翻转等行为.

Wiersma课题组 ${ }^{[40,41]}$ 利用激光直写系统可在亚微 米尺度获得精细的三维交联液晶聚合物结构. 他们 通过特殊设计成功制备了在光驱动下可行走的微米 尺度行走器, 该行走器的主体是交联液晶聚合物材
料, 在532 nm激光作用下可产生 $20 \%$ 的收缩，最大应 力可达 $260 \pm 2 \mathrm{kPa}^{[41]}$. 因为微型行走器尺度过小会导 致其和接触表面间的黏附力变得和“肌肉”产生的应 力可相抗衡, 从而行进难度增加. 他们通过对行走器 腿部进行特殊设计可减小其和接触表面的黏附力, 成功将行走器尺度做到微米级. 在不同的基板表面, 该微型行走器可完成无规行走、旋转、再定位、按轨 迹行走等不同动作. 由于无需特别针对某一部位照 射就可完成移动动作, 理论上该微型行走器可直 接从周围环境吸收光能, 从而完成驱动过程, 如图 10 所示.

\section{3 结语}

由于光响应基团的引人，交联液晶聚合物可在 光的作用下发生丰富的响应性行为. 光致形变交联 液晶聚合物材料在实际的应用中有诸多优点，例如 易于远程操控, 无需任何辅助设备, 通过其自身形变 就可将光能直接转化为机械能等，因此，该类材料获 得了广泛的关注. 但是, 该类材料的发展还存在一些 缺陷, 例如现阶段其能源转换效率和速度仍无法和 光电材料匹敌, 还需进一步提高; 由于制备过程需化 (a)

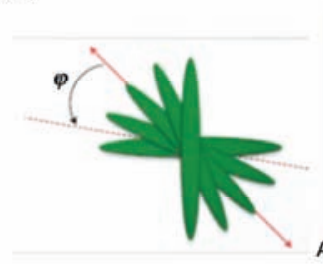

(b)

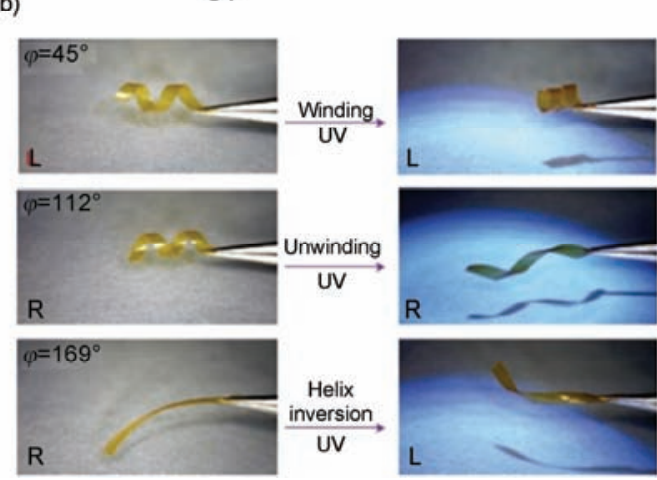

(c)

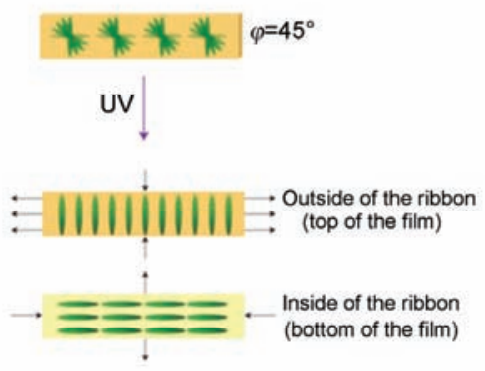

(d)

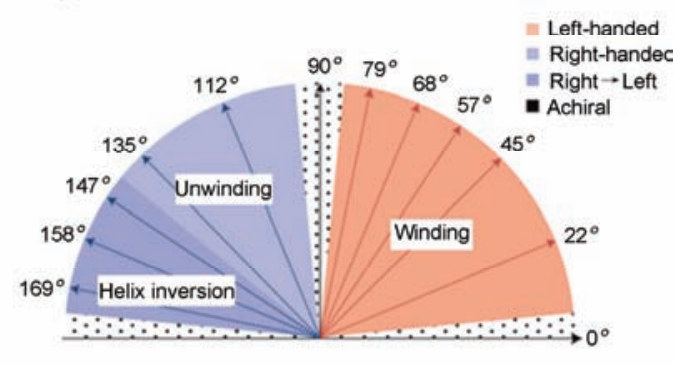

图 9 (网络版彩色)(a) 薄膜内部分子螺旋排列示意图; (b) 不同裁剪角度下得到的丝带不同光致形变方式; (c) 丝带形变原理图, $\varphi=45^{\circ}$; (d) 不 同裁剪角度下丝带形变模式图 ${ }^{[39]}$

Figure 9 (Color online) (a) Molecular organization within the film. (b) Spiral ribbons display isochoric winding, unwinding and helix inversion as dictated by their initial shape and geometry. (c) Scheme to represent the mechanism of the ribbon under irradiation with ultraviolet light for $\varphi=45^{\circ}$. (d) Shape and photoactuation modes of the polymer springs as a function of the angular offset ${ }^{[39]}$ 


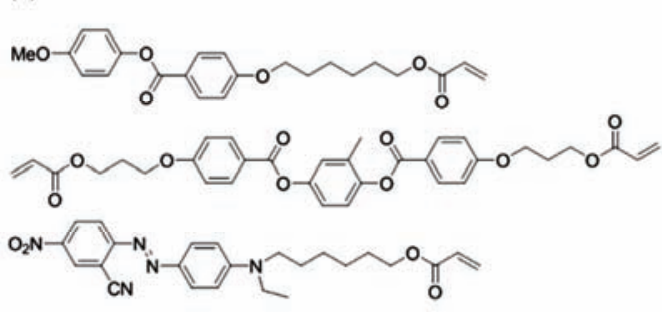

(b)

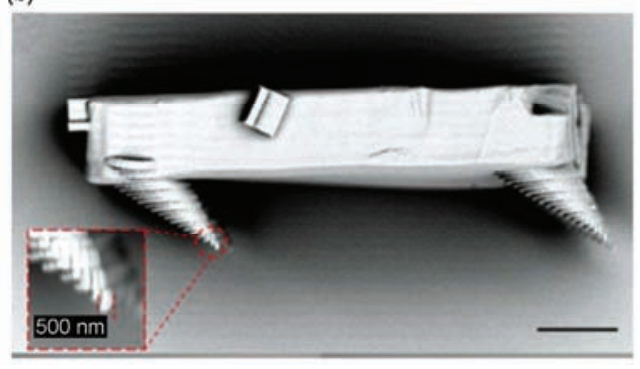

(c)

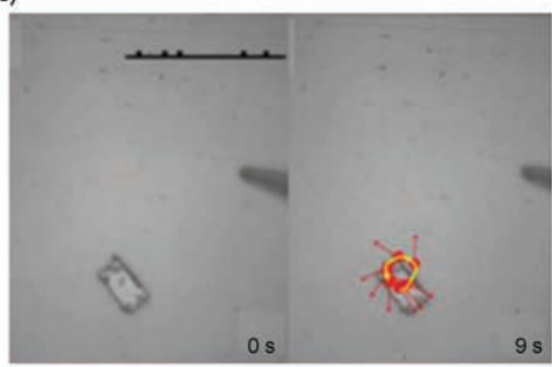

(d)

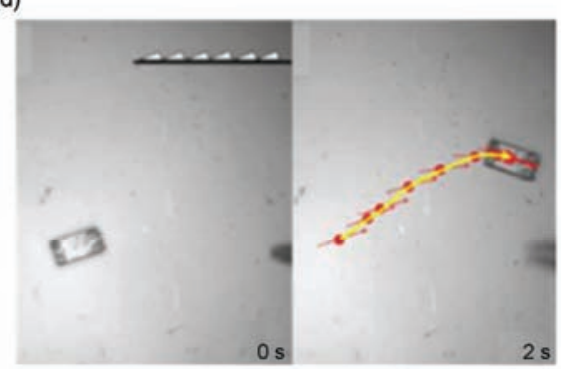

图 10 (网络版彩色)(a) 单体和交联剂化学结构式; (b) 微型行走器扫描电子显微镜照片; (c) 微型行走器在有聚酰亚胺图层的基板上做旋转运 动; (d) 微型行走器在有沟槽的基板上沿一定轨迹行走 ${ }^{[41]}$

Figure 10 (Color online) (a) Molecules used in the experiment. (b) SEM image of the microwalker. (c) Microscopic walker rotates with one leg stuck onto the PI coated surface. (d) Microscopic walker walks in the direction determined by the grating groove pattern ${ }^{[41]}$

学交联，无法利用传统聚合物熔融、溶液等加工方式 实现大批量生产; 受交联液晶聚合物薄膜制备工艺 限制, 难以制备大尺寸样品. 此外, 要想实现产业化 应用, 还要进一步提高交联液晶聚合物的力学性能. 当前执行器的制备过程几乎都局限于简单且单一的
小部件的生产，例如薄膜、丝带等，没有考虑如何集 成到其他系统中，在此过程中还需考虑结构的控制 以及加工工艺条件等. 随着一项项新技术的出现，相 信光致形变交联液晶聚合物及其执行器的研究将朝 着更深更广的领域发展直至走进人们的生活.

\section{参考文献}

1 Finkelmann H. Liquid crystalline polymers. Angew Chem Int Ed, 1987, 26: 816-824

2 Zentel R. Liquid crystalline elastomers. Adv Mater, 1989, 1: 321-329

3 Kelly S M. Anisotropic networks. J Mater Chem, 1995, 5: 2047-2061

4 Ohm C, Brehmer M, Zentel R. Liquid crystalline elastomers as actuators and sensors. Adv Mater, 2010, 22: 3366-3387

5 Ikeda T, Mamiya J, Yu Y L. Photomechanics of liquid-crystalline elastomers and other polymers. Angew Chem Int Ed, 2007, 46: 506-528

6 Bar-Cohen Y, Zhang Q. Electroactive polymer actuators and sensors. MRS Bull, 2008, 33: 173-181

7 Wang W, Wang X Z, Cheng F T, et al. Light-driven soft actuators based on photoresponsive polymer materials (in Chinese). Prog Chem, 2011, 23: 1165-1173 [王威, 王晓振, 程伏涛, 等. 基于光响应高分子材料的柔性执行器件. 化学进展, 2011, 23: 1165-1173]

8 Yu Y, Ikeda T. Soft actuators based on liquid-crystalline elastomers. Angew Chem Int Ed, 2006, 45: 5416-5418

9 Wei J, Yu Y. Photodeformable polymer gels and crosslinked liquid-crystalline polymers. Soft Matter, 2012, 8: 8050-8059

10 Ube T, Ikeda T. Photomobile polymer materials with crosslinked liquid-crystalline structures: Molecular design, fabrication, and functions. Angew Chem Int Ed, 2014, 53: 10290-10299

11 Wu Y, Ikeda T, Zhang Q. Three-dimensional manipulation of an azo polymer liquid crystal with unpolarized light. Adv Mater, 1999, 11: 300-302

12 White T J, Tabiryan N V, Serak S V, et al. A high frequency photodriven polymer oscillator. Soft Matter, 2008, 4: 1796-1798

13 Hrozhyk U, Serak S, Tabiryan N, et al. Bidirectional photoresponse of surface pretreated azobenzene liquid crystal polymer networks. Opt Express, 2009, 17: 716-722 
14 White T J, Serak S V, Tabiryan N V, et al. Polarization-controlled, photodriven bending in monodomain liquid crystal elastomer cantilevers. J Mater Chem, 2009, 19: 9284

15 Serak S, Tabiryan N, Vergara R, et al. Liquid crystalline polymer cantilever oscillators fueled by light. Soft Matter, 2010, 6: 779-783

16 Finkelmann H, Nishikawa E, Pereira G G, et al. A new opto-mechanical effect in solids. Phys Rev Lett, 2001, 87: 015501

17 Li M H, Keller P, Li B, et al. Light-driven side-on nematic elastomer actuators. Adv Mater, 2003, 15: 569-572

18 Ikeda T, Nakano M, Yu Y L, et al. Anisotropic bending and unbending behavior of azobenzene liquid-crystalline gels by light exposure. Adv Mater, 2003, 15: 201-205

19 Yu Y, Nakano M, Shishido A, et al. Effect of cross-linking density on photoinduced bending behavior of oriented liquid-crystalline network films containing azobenzene. Chem Mater, 2004, 16: 1637-1643

20 Yu Y L, Nakano M, Ikeda T. Directed bending of a polymer film by light-miniaturizing a simple photomechanical system could expand its range of applications. Nature, 2003, 425: 145

21 Kondo M, Yu Y L, Ikeda T. How does the initial alignment of mesogens affect the photoinduced bending behavior of liquid-crystalline elastomers? Angew Chem Int Ed, 2006, 45: 1378-1382

22 Yu Y L, Maeda T, Mamiya J, et al. Photomechanical effects of ferroelectric liquid-crystalline elastomers containing azobenzene chromophores. Angew Chem Int Ed, 2007, 46: 881-883

23 Yin R Y, Xu W X, Kondo M, et al. Can sunlight drive the photoinduced bending of polymer films? J Mater Chem, 2009, 19: 3141-3143

24 Cheng F T, Zhang Y Y, Yin R Y, et al. Visible light induced bending and unbending behavior of crosslinked liquid-crystalline polymer films containing azotolane moieties. J Mater Chem, 2010, 20: 4888-4896

$25 \mathrm{Wu}$ W, Yao L M, Yang T S, et al. NIR-light-induced deformation of cross-linked liquid-crystal polymers using upconversion nanophosphors. J Am Chem Soc, 2011, 133: 15810-15813

26 Jiang $\mathrm{Z}, \mathrm{Xu} \mathrm{M}, \mathrm{Li} \mathrm{F} \mathrm{Y,} \mathrm{et} \mathrm{al.} \mathrm{Red-light-controllable} \mathrm{liquid-crystal} \mathrm{soft} \mathrm{actuators} \mathrm{via} \mathrm{low-power} \mathrm{excited} \mathrm{upconversion} \mathrm{based} \mathrm{on}$ triplet-triplet annihilation. J Am Chem Soc, 2013, 135: 16446-16453

27 Liu D Q, Bastiaansen C W M, den Toonder J M J, et al. Photo-switchable surface topologies in chiral nematic coatings. Angew Chem Int Ed, 2012, 51: 892-896

28 Liu D Q, Liu L, Onck P R, et al. Reverse switching of surface roughness in a self-organized polydomain liquid crystal coating. Proc Natl Acad Sci USA, 2015, 112: 3880-3885

29 Liu D Q, Broer D J. Self-assembled dynamic 3D fingerprints in liquid-crystal coatings towards controllable friction and adhesion. Angew Chem Int Ed, 2014, 53: 4542-4546

30 Li C, Cheng F T, Lü J A, et al. Light-controlled quick switch of adhesion on a micro-arrayed liquid crystal polymer superhydrophobic film. Soft Matter, 2012, 8: 3730-3733

31 Yan Z, Ji X M, Wu W, et al. Light-switchable behavior of a microarray of azobenzene liquid crystal polymer induced by photodeformation. Macromol Rapid Commun, 2012, 33: 1362-1367

32 Zhao J Q, Liu Y Y, Yu Y L. Dual-responsive inverse opal films based on a crosslinked liquid crystal polymer containing azobenzene. J Mater Chem C, 2014, 2: 10262-10267

33 Yamada M, Kondo M, Mamiya J I, et al. Photomobile polymer materials: Towards light-driven plastic motors. Angew Chem Int Ed, 2008, 47: 4986-4988

34 Yamada M, Kondo M, Miyasato R, et al. Photomobile polymer materials-various three-dimensional movements. J Mater Chem, 2009, 19: 60-62

35 Cheng F T, Yin R Y, Zhang Y Y, et al. Fully plastic microrobots which manipulate objects using only visible light. Soft Matter, 2010, 6: 3447-3449

36 Chen M L, Xing X, Liu Z, et al. Photodeformable polymer material: Towards light-driven micropump applications. Appl Phys A Mater Sci Proc, 2010, 100: 39-43

37 Chen M, Huang H, Zhu Y, et al. Photodeformable CLCP material: Study on photo-activated microvalve applications. Appl Phys A Mater Sci Proc, 2011, 102: 667-672

38 van Oosten C L, Bastiaansen C W M, Broer D J. Printed artificial cilia from liquid-crystal network actuators modularly driven by light. Nat Mater, 2009, 8: 677-682

39 Iamsaard S, Asshoff S J, Matt B, et al. Conversion of light into macroscopic helical motion. Nat Chem, 2014, 6: 229-235

40 Zeng H, Martella D, Wasylczyk P, et al. High-resolution 3D direct laser writing for liquid-crystalline elastomer microstructures. Adv Mater, 2014, 26: 2319-2322

41 Zeng H, Wasylczyk P, Parmeggiani C, et al. Light-fueled microscopic walkers. Adv Mater, 2015, 27: 3883-3887 


\title{
Photoinduced deformation of crosslinked liquid crystalline polymers and soft actuators
}

\author{
GU Wei ${ }^{1}$, QING Xin ${ }^{1}$, WEI Jia ${ }^{1} \&$ YU YanLei ${ }^{1,2}$ \\ ${ }^{1}$ Department of Materials Science, Fudan University, Shanghai 200433, China; \\ ${ }^{2}$ State Key Laboratory of Molecular Engineering of Polymers, Fudan University, Shanghai 200433, China
}

Photoresponsive polymeric materials are a type of functional materials that can absorb photo energy and undergo intra- or inter-molecular physical or chemical reactions. As a result, the materials exhibit macroscopic property changes when exposed to light, such as shape, color or refractive index. They attract more and more attention for the reason that light is an environmental-friendly and remotely controllable stimulus source. Liquid crystals exhibit cooperative motion of molecules in changing the molecular alignment by external stimuli. That means that only a small amount of energy is needed to bring about the alignment change in the whole system. Crosslinked liquid crystalline polymers (CLCPs) can be prepared by crossliking conventional liquid crystal polymers into a network, which possess both the order of liquid crystals and the elasticity of elastomers. The cooperative effect of liquid crystals coupled with polymer network structure gives rise to the characteristic properties of CLCPs. The initial ordering of mesogens can be fixed by the crosslinkers in CLCPs, which might cause a quick change in shape due to a fast order-disorder transition. CLCPs have been a hot topic because large deformation can be induced by changing the alignment of mesogens in CLCPs by external stimuli such as electric fields, changes in temperature, humidity and light. Incorporation of photochromic moieties into CLCPs provides the materials with photo controllable properties. Photoresponsive CLCPs can generate sophisticated movements including contraction/expansion, bending and twisting which convert photo energy into mechanical energy directly. Therefore, the CLCPs can be fabricated into various soft smart actuators by rational design, which brings about broad applications in artificial muscles, microrobots, microvalves, sensors and so on.

In this review, the photoresponsive mechanism is firstly elucidated involving photochemical phase transition and photo realignment. Then, we detail the historical development of photoresponsive CLCPs, with emphasis on lightdriven mechanical responses concerning two dimensional and three dimensional movements and surface property changes caused by deformation. We also summarize the recent progress of light-driven soft actuators made of photoresponsive CLCPs. We end with an outlook of existing challenges and opportunities.

photoresponsive, crosslinked liquid crystalline polymers, actuators, deformation, azobenzene doi: 10.1360/N972016-00006 